\title{
Prognostic Role of Hepatoma-derived Growth Factor in Solid Tumors of Eastern Asia: a Systematic Review and Meta- Analysis
}

\author{
Ci-Hang Bao ${ }^{1}$, Kun Liu ${ }^{2}$, Xin-Tong Wang ${ }^{1}$, Wei Ma ${ }^{3}$, Jian-Bo Wang ${ }^{1}$, Cong Wang ${ }^{1}$, \\ Yi-Bin Jia ${ }^{1}$, Na-Na Wang ${ }^{1}$, Bing-Xu Tan ${ }^{1}$, Qing-Xu Song ${ }^{1}$, Yu-Feng Cheng ${ }^{1 *}$
}

\begin{abstract}
Hepatoma-derived growth factor (HDGF) is a novel jack-of-all-trades in cancer. Here we quantify the prognostic impact of this biomarker and assess how consistent is its expression in solid tumors. A comprehensive search strategy was used to search relevant literature updated on October 3, 2014 in PubMed, EMBASE and WEB of Science. Correlations between HDGF expression and clinicopathological features or cancer prognosis was analyzed. All pooled HRs or ORs were derived from random-effects models. Twenty-six studies, primarily in Eastern Asia, covering 2,803 patients were included in the analysis, all of them published during the past decade. We found that HDGF overexpression was significantly associated with overall survival $(\mathrm{OS})\left(\mathrm{HR}_{\mathrm{OS}}=\mathbf{2 . 3 5}\right.$, 95\% CI $=2.04-2.71, p<0.001)$ and disease free survival (DFS) $\left(\mathrm{HR}_{\mathrm{DFS}}=\mathbf{2 . 2 5}, \mathbf{9 5 \%} \mathrm{CI}=\mathbf{1 . 8 1}-2.79, p<0.001\right)$ in solid tumors, especially in non-small cell lung cancer, hepatocellular carcinoma and cholangiocarcinoma (CCA). Moreover, multivariate survival analysis showed that HDGF overexpression was an independent predictor of poor prognosis $\left(\mathrm{HR}_{\mathrm{OS}}=\mathbf{2 . 4 1}, 95 \% \mathrm{CI}: \mathbf{2 . 0 2 - 2 . 8 1}, p<0.001 ; \mathrm{HR}_{\mathrm{DFS}}=\mathbf{2 . 3 9}, \mathbf{9 5 \%} \mathrm{CI}: \mathbf{1 . 7 7 - 3 . 2 4}, p<0.001\right)$. In addition, HDGF overexpression was significantly associated with tumor category (T3-4 versus T1-2, OR=2.12, 95\% CI: 1.17-3.83, $p=0.013$ ) and lymph node status $(\mathrm{N}+$ versus $\mathrm{N}-, \mathrm{OR}=\mathbf{2 . 3 7 , 9 5 \%} \mathrm{CI}: \mathbf{1 . 3 1 - 4 . 2 9}, p=0.03)$ in CCA. This study provides a comprehensive examination of the literature available on the association of HDGF overexpression with OS, DFS and some clinicopathological features in solid tumors. Meta-analysis results provide evidence that HDGF may be a new indicator of poor cancer prognosis. Considering the limitations of the eligible studies, other large-scale prospective trials must be conducted to clarify the prognostic value of HDGF in predicting cancer survival.
\end{abstract}

Keywords: HDGF - prognosis - clinicopathological features - solid tumors - meta-analysis

Asian Pac J Cancer Prev, 16 (5), 1803-1811

\section{Introduction}

GLOBOCAN 2012 showed that the new cases and deaths of cancers were respectively increased by $11.02 \%$ and $1.08 \%$ in 2012 compared with 2008. Despite the introduction of hundreds of new anticancer drugs, the consensus is that, for most forms of cancer, enduring disease-free responses are rare, and cures even rarer (Hanahan, 2014). The clinical outcome is disillusionary (Woodward, 2014) mainly due to tumor local recurrence or distal metastasis (Ordonez-Moran et al., 2014). Thus, it is still emergency and necessary for us to find more effective and applicable tumor markers to predict prognosis and progression of cancers and then improve the patients' quality of life and reduce mortality.

In recent years, elevated levels of hepatoma-derived growth factor (HDGF) have been observed in a variety of malignancies and high levels of HDGF appears to be associated with increased malignancy across cancers, as witnessed by the correlation with adverse characteristics such as poor patient survival (Hu et al., 2003; Ren et al., 2004; Iwasaki et al., 2005; Uyama et al., 2006; Yamamoto et al., 2006; Yoshida et al., 2006; Chang et al., 2007; Yamamoto et al., 2007; Zhou et al., 2007a; Hu et al., 2009; Savola et al., 2009; Zhang et al., 2010; Zhou et al., 2010b; Liu et al., 2011; Wang et al., 2011; Ye et al., 2011; Zhang et al., 2011; Chen et al., 2012a; Chen et al., 2012b; Hsu et al., 2012; Lin et al., 2012; Han et al., 2013; Hanada et al., 2013; Li et al., 2013a; Li et al., 2013b; Li et al., 2013c; Yang et al., 2013; Guo et al., 2014b; Song et al., 2014; Wang et al., 2014; Yang et al., 2014; Zhang et al., 2014). As a multifunctional protein (Zhao et al., 2011; Bao et al., 2014) widely expressed by many types of human cells, HDGF is involved in the regulations of a myriad of cancer

${ }^{1}$ Department of Radiation Oncology, Qilu Hospital of Shandong University, ${ }^{2}$ Center for Health Management and Policy, Key Lab of Health Economics and Policy, Ministry of Health, Shandong University, Jinan, ${ }^{3}$ Department of Radiation Oncology, Cancer Hospital, General Hospital of Ningxia Medical University, Yinchuan, China*For correspondence: qlcyf2014new@163.com 
cell activities during cancer transformation (Yang et al., 2013), apoptosis (Zhao et al., 2011), angiogenesis(Ren et al., 2009), and metastasis (Chen et al., 2012a). Therefore, HDGF not only promote cancer progression but also may be an indicator of the prognosis of various tumors.

To the best of our knowledge, no meta-analysis data on the correlation of HDGF expression with the prognosis and survival of patients with whole solid tumors are yet available. Thus, we conducted a meta-analysis aimed at evaluating the value of HDGF as a prognostic marker for cancer and to determine the relationship between HDGF and several clinicpathological features of solid tumors.

\section{Materials and Methods}

\section{Data sources and searches}

The meta-analysis was performed in accordance with the guidelines of Preferred Reporting Items for Systematic Reviews and Meta-Analyses. We conducted systematic computerized searches in the PubMed and EMBASE databases as well as WEB of Science to identify all published articles on the prognostic function of HDGF expression in tumor tissues. We used combinations of the following search terms: "HDGF or hepatomaderived growth factor", "tumor or cancer or carcinoma or malignant or neoplasm", "prognosis or prognostic or survival or outcome". The final search was updated on October 3, 2014.

\section{Study selection}

The studies included in the meta-analysis met all of the following inclusion criteria: $(i)$ evaluated the correlation between immunohistochemistry (IHC)detected HDGF expression and overall survival (OS) and disease free survival (DFS) in solid tumors; ( $i$ i) used a cohort design; (iii) directly extracted the hazard ratio (HR) and 95\% confidence interval (CI) from an original article or provided sufficient data for the HR and 95\%CI calculations; and (iv) sample size was equal to or greater than 50. Studies considered ineligible for the meta-analysis were as follows: review articles, conference abstracts, editorials, thesis or case reports; studies in which clinical endpoints other than the OS and DFS were used; and articles with insufficient published data for estimating the $\mathrm{HR}$ and $95 \% \mathrm{CI}$. For multiple publications from the same institution with identical or overlapping patient cohorts, only the largest series was included in the analysis to avoid duplicate information. The study identification and selection processes were presented in Figure 1.

\section{Data extraction and quality assessment}

The final articles included were assessed independently by two reviewers (Bao and Liu) (Table 1). Standardized abstraction sheets were used to record data from individual studies. Data retrieved from the first author, year of publication, age, follow-up time, source, cancer types, number of analyzed patients, gender, language, blinded HDGF measurements, cutoff scores, analysis of variable, HR estimation for OS or DFS, subcellular localization, prognosis value and Newcastle Ottawa Scale (NOS) score. For each study, the HR was estimated using the method reported by Parmar et al. (1998). HRs were extracted preferentially from multivariable analyses where available. Otherwise, HRs from univariate analyses were extracted. HR estimates and 95\%CIs were either directly obtained from the original article or calculated using parameters such as statistics of observed minus expected events and variance provided in the papers. Otherwise, the number of patients at risk in each group, as well as the number of events and $\mathrm{P}$ value of the log-rank statistic, was retrieved to allow approximate calculation of the HR estimate and its variance. If the study did not provide the HR but reported the survival curve, survival rates at specified times were extracted to reconstruct the HR estimate and its variance, with the assumption that the rate of patients censored was constant during the follow-up (Tierney et al., 2007). Survival rates on the graphical representation of the survival curves were read by Engauge Digitizer version 2.5 (Cao et al., 2013). Quality assessment of the cohort studies was performed using the NOS (Wells et al., 2012). Given the variability in the quality of cohort studies found in our initial literature search, we considered studies as of high quality if they achieved a score of six or more.

\section{Data synthesis}

The meta-analysis was conducted initially for all included studies for each of the endpoints of interest. Subgroup analyses were conducted for predefined parameters such as subcellular localization, cancer type, cases, cutoff, whether data were derived from univariate or multivariable analyses. Cancer type subgroups were generated for the main outcome if at least two studies or three datasets on that site were available; the remaining studies were pooled in a subgroup termed "others."

\section{Statistical analysis}

Combined OR and 95\%CI were used to assess the strength of association of HDGF expression with clinicpathological features of cancer, $p<0.05$ indicated statistical significance. Estimates of OR were weighted

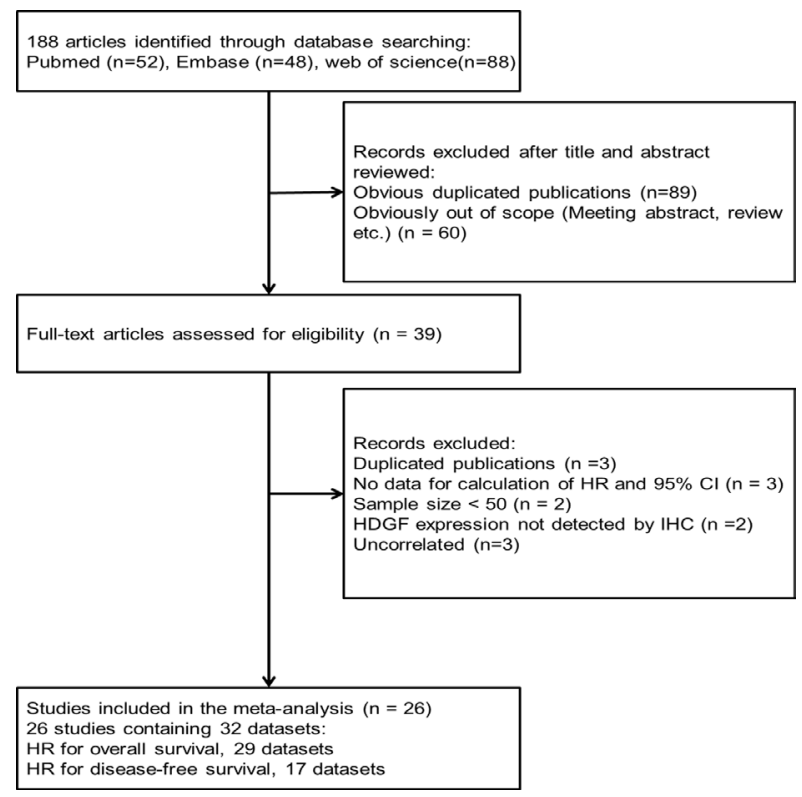

Figure 1. Flow Diagram of the Study Selection Process in the Meta-analysis 
and pooled using the Mantel-Haenszel and random-effect model. Combined HR and 95\% CI were used to assess the strength of association of HDGF expression with OS or DFS; HR>1 indicated poor prognosis of patients with HDGF expression if the 95\%CI exceeded 1 . The statistical significance of the pooled HR was determined by a Z-test. $p<0.05$ indicated statistical significance. Heterogeneity was assessed using $\mathrm{I}^{2}$ and $\mathrm{Q}$ statistics. For the $\mathrm{I}^{2}$ statistic, heterogeneity was interpreted as absent $\left(\mathrm{I}^{2}<25 \%\right)$, moderate $\left(\mathrm{I}^{2}=25 \%-50 \%\right)$, or extreme $\left(\mathrm{I}^{2}=50 \%-100 \%\right)$ (Higgins et al., 2003). For the $\mathrm{Q}$ statistic, $p<0.10$ was considered statistically significant for heterogeneity. Estimates of HR were weighted and pooled using the generic inversevariance and random-effect model. Subgroup analysis was conducted to evaluate the effect of a potential factor on the association as a source of heterogeneity. Analyses were conducted for all studies, and differences between the subgroups were assessed using methods described by Deeks et al. (2011). To validate the robustness of the meta-analysis findings, sensitivity analysis was performed by sequentially omitting each individual study using the "metainf" STATA command. Potential publication bias was evaluated using Begg's and Egger's asymmetry tests (Egger et al., 1997) and through visual inspection of funnel plots, in which the standard error was plotted against $\log (\mathrm{HR})$ to form a simple scatterplot. When the statistical significance of Egger's test results was defined at $p<0.10$, we conducted a trim and fill analysis(Duval et al., 2000), which yields an effect adjusted for funnel plot asymmetry. The meta-analysis was performed using STATA version 11 (StataCorp LP, College Station, TX, USA). All P values were two tailed.

\section{Results}

\section{Study inclusion and characteristics}

The literature search identified 188 potentially relevant articles. Of these articles, 60 were excluded based on the titles and abstracts because of obvious lack of relevance, and 89 were excluded because of obvious duplicated publications. The following articles were also excluded: three duplicated publications (Zhou et al., 2007b; Zhou et al., 2010a; Yang et al., 2014); three without data for $\mathrm{HR}$ and 95\%CI calculations and adequate contact with the investigators could not be established (Natrajan et al., 2006; Mao et al., 2008; Hanada et al., 2013); two with a sample size<50 (Hsu et al., 2012; Tao et al., 2014); two in which HDGF expression was not detected by IHC methods (Savola et al., 2009; Zhang et al., 2010); three uncorrelated. Finally, 26 cohort studies were found eligible for the meta-analysis (Hu et al., 2003; Ren et al., 2004; Iwasaki et al., 2005; Uyama et al., 2006; Yamamoto et al., 2006; Yoshida et al., 2006; Chang et al., 2007; Yamamoto et al., 2007; Zhou et al., 2007a; Hu et al., 2009; Zhou et al., 2010b; Liu et al., 2011; Wang et al., 2011; Zhang et al., 2011; Chen et al., 2012a; Chen et al., 2012b; Lin et al., 2012; Han et al., 2013; Li et al., 2013a; Li et al., 2013b; Li et al., 2013c; Yang et al., 2013; Guo et al., 2014b; Song et al., 2014; Wang et al., 2014; Zhang et al., 2014), containing 32 datasets (29 datasets for OS; 17 datasets for DFS) were qualified to the standard of the meta-analysis. The main characteristics of the selected studies were summarized in Table 1 . The 26 cohort studies, which involved 2,803 cancer patients with 50 to 317 patients per study, were primarily conducted in Eastern Asia and published between 2003 and 2014. In addition, the expression of HDGF was detected by IHC in all studies. The HR estimations in 27 datasets were directly extracted from original data, and five were extrapolated from survival curves. Information on the specified cutoff (5\% or at least moderate staining) for HDGF expression could be obtained from all enrolled studies. All of the 26 studies identified HDGF expression as an indicator of poor prognosis. Most studies had established HDGF to be an independent prognostic factor in solid tumors (Table 1). According to the quality criteria, all cohort studies were of high quality and had scores of six or more.

\section{Correlation of HDGF expression with clinicopathological parameters}

From the biological point of view it is very difficult to compare and summarize data from different tumor features, especially when dealing with TNM classifications. In the manuscript (data not show), this is reflected by the high heterogeneity when comparing the expression of HDGF in solid tumors with pathological data. It would be of importance to analyse the distinct tumor enteties by themselves. However, only cholangiocarcinoma (CCA) has enough data to be analysed. HDGF expression was not associated with certain clinical parameters of CCA, such as gender (Male versus Female, pooled $\mathrm{OR}=0.78,95 \% \mathrm{CI}$ : $0.23-2.60, p=0.684$ and $\mathrm{I}^{2}=75.8$ ), TNM stage (III-IV versus

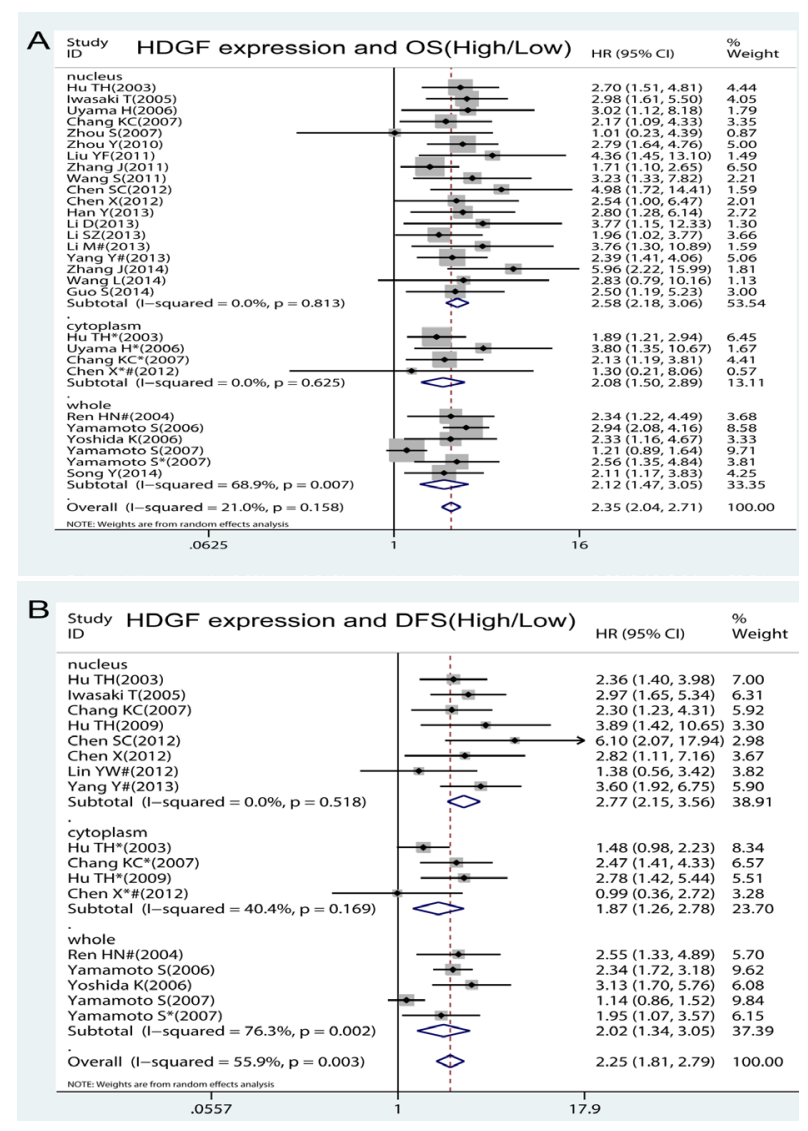

Figure 2. Forest Plot of HRs for the OS Rate (A) and DFS Rate (B) among the Included Studies 
Table 2. HDGF Overexpression and Clinicopathological Features in CCA Patients

\begin{tabular}{|c|c|c|c|c|c|c|c|c|}
\hline $\begin{array}{l}\text { First author } \\
\text { (Year) }\end{array}$ & Source & $\begin{array}{l}\text { Cancer } \\
\text { Type }\end{array}$ & Cases & $\begin{array}{c}\text { T category (T1-2/ } \\
\text { 3-4: high/low) }\end{array}$ & $\begin{array}{l}\text { Ncategory }(\mathrm{N}-/ \\
\mathrm{N}+\text { : high/low) }\end{array}$ & $\begin{array}{l}\text { TNM (I-II/III-IV: } \\
\text { high/low) }\end{array}$ & $\begin{array}{c}\text { Gender }(\mathrm{F} / \mathrm{M}: \\
\text { high/low })\end{array}$ & $\begin{array}{l}\text { Differentiation } \\
\text { (high/low) }\end{array}$ \\
\hline $\begin{array}{l}\text { Liu YF } \\
(2011)\end{array}$ & China & $\mathrm{HC}$ & 58 & $\begin{array}{l}\text { T1-2: } 12 / 21 \\
\text { T3-4: } 15 / 10\end{array}$ & $\begin{array}{l}\mathrm{N}-: 16 / 23 \\
\mathrm{~N}+: 11 / 8\end{array}$ & $\begin{array}{l}\text { I-II: } 11 / 16 \\
\text { III-IV: } 17 / 14\end{array}$ & $\begin{array}{l}\text { F: } 12 / 6 \\
\text { M: } 15 / 25\end{array}$ & $\begin{array}{l}\text { W/M:16/18 } \\
\text { P/U:12/12 }\end{array}$ \\
\hline $\begin{array}{l}\text { Han Y } \\
(2013)\end{array}$ & China & EHCC & 65 & $\begin{array}{l}\text { T1-2: } 16 / 24 \\
\text { T3-4: } 14 / 11\end{array}$ & $\begin{array}{l}\mathrm{N}-: 12 / 21 \\
\mathrm{~N}+: 18 / 14\end{array}$ & $\mathrm{NR}$ & $\begin{array}{l}\text { F: } 12 / 10 \\
\text { M: } 18 / 25\end{array}$ & $\begin{array}{l}\text { W/M:8/19 } \\
\text { P/U:22/16 }\end{array}$ \\
\hline $\begin{array}{l}\text { Guo S } \\
(2014) \\
\end{array}$ & China & IHCC & 83 & $\begin{array}{l}\text { T1-2: } 11 / 12 \\
\text { T3-4: } 32 / 28\end{array}$ & $\begin{array}{l}\mathrm{N}-: 25 / 32 \\
\mathrm{~N}+: 18 / 8\end{array}$ & $\begin{array}{l}\text { I-II: } 18 / 23 \\
\text { III-IV: } 24 / 18\end{array}$ & $\begin{array}{l}\text { F: } 16 / 23 \\
\text { M: } 27 / 17\end{array}$ & $\begin{array}{l}\text { W/M:29/29 } \\
\text { P/U:14/14 }\end{array}$ \\
\hline
\end{tabular}

Meta-analysis of HDGF Overexpression and Clinicopathological Features in CCA Patients

\begin{tabular}{lcccccc}
\hline Categories & Studies (cases) & OR(95\% CI) & $\mathrm{I}^{2}(\%)$ & $\mathrm{Ph}$ & $\mathrm{Z}$ & $\mathrm{P}$ \\
\hline Gender (Male vs Female) & $3(206)$ & $0.78(0.23,2.59)$ & 75.8 & 0.016 & 0.41 & 0.684 \\
Tumor category (T3-4 vs T1-2) & $3(206)$ & $2.12(1.17,3.83)$ & 0 & 0.894 & 2.48 & 0.013 \\
Lymph node status (N+ vs N-) & $3(206)$ & $2.37(1.31,4.29)$ & 0 & 0.876 & 2.86 & 0.004 \\
TNM stage (III-IV vs I-II) & $2(141)$ & $1.73(0.89,3.37)$ & 0 & 0.959 & 1.61 & 0.108 \\
Differentiation (P/U vs W/M) & $3(206)$ & $1.50(0.73,3.10)$ & 37.2 & 0.204 & 1.1 & 0.272 \\
\hline
\end{tabular}

Note: CCA cholangiocarcinoma; HC Hilar cholangiocarcinoma; EHCC Extrahepatic cholangiocarcinoma; IHCC intrahepatic cholangiocarcinoma Well/moderately=W/M; Poorly/undifferentiated=P/U.Ph denotes $\mathrm{P}$ value for heterogeneity based on Q test; $\mathrm{P}$ denotes $\mathrm{P}$ value for statistical significance based on $\mathrm{Z}$ test; vs, versus; *All pooled ORs were derived from random-effects modelt

I-II, pooled OR=1.73, 95\%CI: $0.89-3.37, p=0.108$ and $\mathrm{I}^{2}=0$ ) and Differentiation(Poorly/undifferentiated versus Well/moderately, pooled $\mathrm{OR}=1.50,95 \% \mathrm{CI}$ : 0.73-3.10, $p=0.272$ and $\mathrm{I}^{2}=37.2$ ). However, cancers with HDGF expression were associated with tumor category (T3-4 versus T1-2, pooled $\mathrm{OR}=2.12,95 \% \mathrm{CI}: 1.17-3.83, p=0.013$ and $\left.\mathrm{I}^{2}=0\right)$ and lymph node status $(\mathrm{N}+$ versus $\mathrm{N}-$, pooled $\mathrm{OR}=2.37,95 \% \mathrm{CI}: 1.31-4.29, p=0.03$ and $\left.\mathrm{I}^{2}=0\right)$. The pooled ORs and their $95 \%$ CIs were presented in detail in Table 2.

Correlation of HDGF with overall survival and disease free survival

We conducted a meta-analysis on the association between HDGF overexpression and the OS of human solid tumors. The pooled HRs and their $95 \%$ CIs were presented in detail in Table 3 . Among the 29 datasets comprising 3,133 patients reported HR for OS, poor prognosis was demonstrated in the pooled HR estimate $\left(\mathrm{HR}=2.35\right.$; 95\%CI, 2.04-2.71, $p<0.001$ and $\left.\mathrm{I}^{2}=21\right)$. It showed that an absent degree of heterogeneity was found in all of the eligible studies. Four of the eligible 29 datasets (14.29\%) reported a non-statistically significant HR (ie, the $95 \%$ confidence intervals crossed 1); a forest plot of all studies was presented as Figure 2A. When the eligible studies were stratified by cases, the combined HRs of cases $(<100)$ and cases $(\geq 100)$ were 2.79 (95\%CI, 2.20$3.55, p<0.001)$ and 2.21 (95\%CI, 1.84-2.67, $p<0.001)$. Differences between cases subgroups were statistically significant (P for subgroup difference, $\mathrm{Psub}=0.041$ ).

When grouped according to the subcellular localization of HDGF protein in studies (Figure 2A), the combined HRs of nucleus, cytoplasm and whole were $2.58(95 \% \mathrm{CI}$, 2.18-3.06, $p<0.001), 2.08$ (95\%CI, 1.50-2.89, $p<0.001)$ and $2.12(95 \% \mathrm{CI}, 1.47-3.05, p<0.001)$, respectively. Although high HDGF for subjects with nucleus was associated with a numerically higher value for the HR than for subjects with cytoplasm and whole, this difference was not statistically significant (Psub=0.084).

In the subgroup analysis (Table 3 ) based on cancer types, the negative prognostic role of high HDGF expression was observed in non-small-cell lung cancer (NSCLC) (HR 2.40, 95\% CI 1.56-3.69, $p<0.001$ ), breast cancer (HR 3.01, 95\% CI 1.56-5.79, $p=0.001$ ), digestion system cancers (HR 2.36, 95\%CI 1.92-2.91, $p<0.001$ ). Differences between cancer types subgroups were not statistically significant $(P s u b=0.776)$. Furthermore, we also studied the pooled risks of HDGF for OS based on tumor types in cancers of the digestion system. We found that the negative prognostic role of high HDGF expression was also observed in CCA (HR 2.91, 95\% CI 1.79-4.71, $p<0.001)$ and hepatocellular carcinoma (HCC) (HR 2.33,95\%CI 1.78-3.06, $p<0.001)$. Furthermore, when the analysis of variable and cutoff were considered, the results did also not show significant changes.

Moreover, a similar difference was also observed in the DFS (Figure 2B, HR=2.25, 95\% CI=1.81-2.79, $p<0.001$ and $\left.\mathrm{I}^{2}=55.6\right)$. It showed that an extreme degree of heterogeneity was found in all of the eligible studies. When grouped according to the subcellular localization of HDGF protein in studies (Figure 2B), the combined HRs of nucleus, cytoplasm and whole were 2.77 (95\%CI, 2.15-3.56, $p<0.001), 1.87(95 \% \mathrm{CI}, 1.26-2.78, p=0.002)$ and 2.03 (95\%CI, 1.34-3.05, $p=0.001)$, respectively. Differences between subgroups were statistically significant (Psub=0.016). When the eligible studies were stratified by HDGF cutoff, the combined HRs of median, $90 \%$ staining, ROC curve and others were $2.79(95 \% \mathrm{CI}$, 2.08-3.75, $p<0.001), 1.89$ (95\%CI, 1.26-2.82, $p=0.002)$, $3.01(95 \% \mathrm{CI}, 2.04-4.44, p<0.001)$ and $1.73(95 \% \mathrm{CI}$, $1.22-2.45, p=0.002)$. Differences between subgroups were statistically significant $(\mathrm{Psub}=0.006)$. Furthermore, when the analysis of variable, cases and cancer types were considered, the results did not show significant changes.

\section{Publication bias analysis and Sensitivity analysis}

Begg's tests indicated that there was no obvious evidence of significant publication bias for the studies included in our meta-analysis $\left(\mathrm{P}_{\mathrm{OS}}=0.048-1.000\right.$ and $\mathrm{P}_{\text {DFS }}=0.012-1.000$, respectively). But Egger's test 
Table 3. Subgroup Analysis and Publication Bias Analysis of Main Outcome*

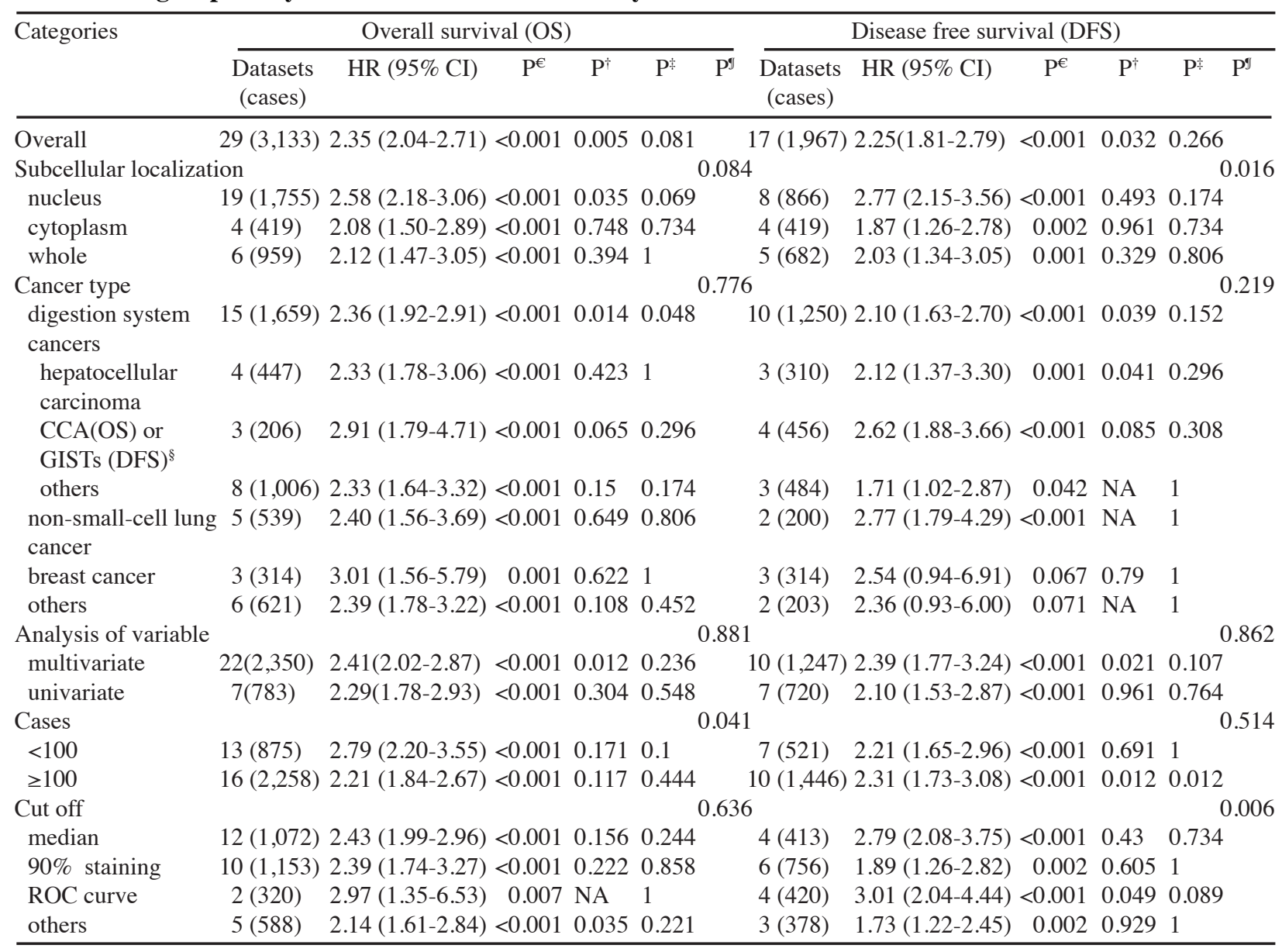

Note: All pooled HRs were derived from random-effects model; All statistical tests were two-sided; CCA, cholangiocarcinoma; GISTs, Gastrointestinal stromal tumors; $\mathrm{CI}=$ confidence interval; $\mathrm{HR}=$ hazard ratio; $\mathrm{NA}=$ not applicable because only two studies; ${ }^{*}$ Subgroup differences were analyzed as described by Deeks et al (42). ${ }^{\circledR} \mathrm{CCA}$ (the subgroup of OS); GISTs (the subgroup of DFS). ${ }^{€} \mathrm{P}$ value for statistical significance based on Z test. "P for Egger's test. "P for Begg's test. "P for subgroup difference

A)

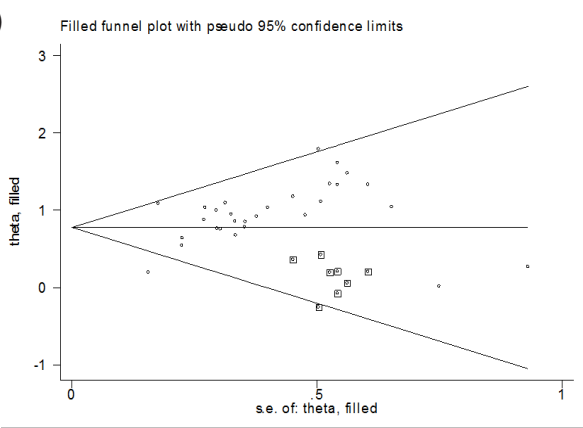

B)

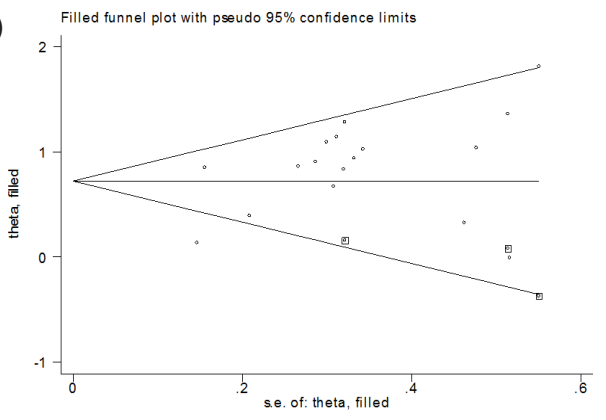

Figure 3. Trim and Fill Results of the OS Rate (A) and DFS Rate (B)

demonstrated a publication bias among the studies regarding $\mathrm{HR}$ of OS and DFS with a $\mathrm{P}$ value of 0.005 and $0.032(p<0.10)$, respectively (Table 3$)$. The funnel plot also revealed an apparent asymmetry that suggested the presence of a potential publication bias (Table 3). Therefore we performed the "trim and fill" analysis. It was estimated that eight studies evaluating the prognostic value of HDGF in OS and three studies evaluating the role of HDGF in DFS remained unpublished. The filled metaanalysis concerning OS (HR=2.17, 95\%CI: $1.89-2.50$, $p<0.001)$ and DFS (HR=2.06, 95\%CI: $1.67-2.54, p<0.001)$ uphelded our pooled results (Figure 3). Moreover, in order to gauge results stability, a sensitivity analysis, in which one study was deleted at a time, was performed. Both of the corresponding pooled ORs and HRs were not significantly changed, suggesting the robustness of our results.

\section{Discussion}

Following the discovery of the prognostic value of high HDGF expression in HCC (Hu et al., 2003), HDGF overexpression and changes of sub- and inter-cellular localization are commonly seen in various types of human cancers. To our knowledge, this present meta-analysis is the first study to systematically evaluate the association between HDGF and clinicopathological features and prognostic factors in solid tumors. In our study, a combined analysis of 26 clinical studies, which detected the HDGF antigen in whole tissue sections, revealed 
a poor prognostic outcome in patients expressing high levels of HDGF.

This meta-analysis showed that elevated HDGF expression did indeed predict negative survival in cancer patients. When grouped according to the subcellular localization of HDGF protein in studies with OS or DFS, we found that HDGF was suggested to be a valid poor prognostic indicator in cancers regardless of its subcellular localization. Although our study also showed that high HDGF estimated by both nucleus and cytoplasm positive had a prognostic value in patients with certain kinds of cancers, strong nuclear staining with minimal cytoplasmic staining was observed in most cases. These results told that up-regulation of nucleus HDGF might be the better evaluation criterion than cytoplasmic HDGF to predict poor prognostic outcome in patients with cancers. Certainly, the conclusion was somewhat faint because of the difference in HDGF cut-off definition. When the eligible studies with DFS were stratified by HDGF cutoff, differences between cases subgroups were statistically significant (Psub=0.006). An explicit definition should be made about the cut-off value of HDGF level for increased survival risk. To date, most researchers use median value or $90 \%$ staining in their laboratory or hospital as the cutoff value and the accurate value were different. Although ROC curve cutoff for high HDGF has highest HR value, the lack of abundant HDGF expression data detected by ROC curve in global population makes it difficult to set a standard cut-off. Thus, it remains to be further explored the HDGF cut-off definition and the relationship between the cytoplasmic HDGF and survival in carcinomas.

In the subgroup analysis, our results indicated that HDGF was associated with the OS or DFS of NSCLC, breast cancer and cancers of the digestion system. Furthermore, we also studied the pooled risks of HDGF for OS or DFS based on tumor types in cancers of the digestion system. We found that the negative prognostic role of high HDGF expression was also observed in CCA, GISTs and HCC. And significant difference was observed in all subgroups $(p<0.05)$. Empirically, HR $>2$ was considered strongly predictive (Hayes et al., 2001). And these different cancer subgroups' results were powerful, though limited studies were included for OS or DFS meta-analysis, respectively. When the eligible studies with OS were grouped by cases, difference between cases subgroups was statistically significant ( $\mathrm{P}$ for subgroup difference $=0.041$ ). Therefore, considering the limitations of the eligible studies in certain cancer, other largescale prospective trials must be conducted to clarify the prognostic value of HDGF in predicting cancer survival.

What makes HDGF account for the poor prognosis in solid tumors? Metastasis is the major cause of death in patients with cancer. Down-regulation of HDGF participates in miRNAs-induced suppression of migration and invasion on several cancer cells (Chen et al., 2014; Guo et al., 2014a), and induces relapse of various cancer cells (Guo et al., 2011). Moreover, previous studies had shown that HDGF overexpression promoted epithelial-mesenchymal transition (Chen et al., 2012a; Tsai et al., 2013) and affected cell metastasis processes via mitogen-activated protein kinase signaling pathways(Mao et al., 2008) or a novel HDGF-disintegrin and metalloproteinase-9 pathway (Zhang et al., 2014), thereby promoting the invasion and metastasis in several cancer cells. In addition, combining the anti-HDGF antibody with anti-VEGF antibody might enhance their antiangiogenic activities(Ren et al., 2009). These qualities thus serve as a potential target for cancer therapy (Yamamoto et al., 2007; Zhou et al., 2010b; Hsu et al., 2012; Li et al., 2013b) and a marker for poor prognosis and metastasis of cancers (Bao et al., 2014). In our study, we also found that HDGF expression was linked to tumor category and lymph node status in CCA. Importantly, multivariate survival analysis showed that HDGF overexpression was an independent predictor of poor prognosis $\left(\mathrm{HR}_{\mathrm{OS}}=2.41,95 \% \mathrm{CI}: 2.02-2.87, p<0.001\right.$; $\mathrm{HR}_{\mathrm{DFS}}=2.39$, 95\%CI: 1.77-3.24, $p<0.001$ ) (Table 3). We infer that in the case of solid tumors, a HDGF inhibitor could improve survival and prognosis. Interestingly, Yamamoto, S. et al previously showed that HDGF expression was an independent prognostic factor for patients with early (pT1-2) stage of the disease, but not for those with advanced (pT3-4) stage (Yamamoto et al., 2007). Several cancers tissue sections in studies were obtained from patients with early-stage cancers (Ren et al., 2004; Zhou et al., 2007a), these indicated that HDGF might be serviceable to predict poor prognostic outcome in patients with early-stage cancers.

Similar to other systematic reviews and meta-analyses, some limitations and strengths of this meta-analysis should be acknowledged. First, marked heterogeneity of subjects existed in DFS. The heterogeneity of the population was probably due to the difference in the baseline characteristics of patients (tumor stage), the cancer type, the cut-off value of HDGF and others. For example, for DFS studies, when we stratified them according to subcellular localization, heterogeneity disappeared in nucleus subgroup $(\mathrm{Ph}=0.518)$. Because such differences might have a residual confounding effect within these studies, we attempted to minimize the effect by using a random effect model. Second, Egger's test demonstrated a publication bias among the studies regarding $\mathrm{HR}$ of OS and DFS with a P value of 0.005 and $0.032(p<0.10)$, respectively (Table 3 ). Obviously, it is unavoidable to miss some data because of unpublished studies. Missing information may reflect a negative or more conservative correlation between HDGF and survival, which could lower the significance of HDGF expression as a predictor of mortality. However, the followed "trim and fill" analysis reinforced the prognostic role of HDGF in cancers patients (Figure 3). Thirdly, there still might be a little error when the approximate calculation method was used to estimate the HR values, although 3 investigators calculated them separately. Fourthly, the studies used various IHC protocols (different kinds of HDGF antibody, dilutions of antibodies etc.) and experimental protocols could have interfered with the results. An explicit definition should be made about the cut-off value of HDGF level for increased survival risk. Finally, tissues are more widely used in HDGF study. However, circulating markers are more acceptable than tissue markers because they can be assayed before surgery and be monitored throughout the 


\section{Ci-Hang Bao et al}

life. The intriguing point is that high levels of HDGF could also be detected in patient serum of lung cancer and extra hepatic CCA (Zhang et al., 2010; Han et al., 2013). This raises the possibility that adverse HDGF levels can be detected in blood samples in other cancer forms as well. More studies should be conducted in future to evaluate the prognostic value of HDGF level in serum. For routine clinical application in the future, the above-mentioned design criterion should be more convincible.

In summary, overexpression of HDGF is significantly associated with several clinicopathological features in CCA and indicated poor OS and DFS of several cancers. The findings suggest that HDGF is a promising prognostic factor for solid tumors, especially in NSCLC, HCC and $\mathrm{CCA}$, and predicts cancer progression such as tumor category and lymph node status in CCA. Considering the limitations of the eligible studies, other large-scale prospective trials must be conducted to clarify the prognostic value of HDGF in predicting cancer survival.

\section{References}

Bao C, Wang J, Ma W, et al (2014). HDGF: a novel jack-of-alltrades in cancer. Future Oncol, 10, 2675-85.

Cao Z, Bao M, Miele L, et al (2013). Tumour vasculogenic mimicry is associated with poor prognosis of human cancer patients: a systemic review and meta-analysis. Eur J Cancer, 49, 3914-23.

Chang KC, Tai MH, Lin JW, et al (2007). Hepatoma-derived growth factor is a novel prognostic factor for gastrointestinal stromal tumors. Int J Cancer, 121, 1059-65.

Chen B, Huang T, Jiang J, et al (2014). miR-141 suppresses proliferation and motility of gastric cancer cells by targeting HDGF. Mol Cell Biochem, 388, 211-8.

Chen SC, Kung ML, Hu TH, et al (2012a). Hepatoma-derived growth factor regulates breast cancer cell invasion by modulating epithelial-mesenchymal transition. J Pathol, 228, 158-69.

Chen X, Yun J, Fei F, et al (2012b). Prognostic value of nuclear hepatoma-derived growth factor (HDGF) localization in patients with breast cancer. Pathol Res Pract, 208, 437-43.

Deeks JJ, Higgins JPT, Altman DG (2011). Chapter 9: analysing data and undertaking meta-analyses. In: Higgins JPT, Green S (eds). Cochrane handbook for systematic reviews of interventions version 5.1.0. [Online]. the cochrane collaboration. http://www.cochrane-handbook.org/.

Duval S, Tweedie R (2000). Trim and fill: A simple funnel-plotbased method of testing and adjusting for publication bias in meta-analysis. Biometrics, 56, 455-63.

Egger M, Davey Smith G, Schneider M, et al (1997). Bias in meta-analysis detected by a simple, graphical test. $B M J$, 315, 629-34.

Guo H, Li W, Zheng T, et al (2014a). MiR-195 targets HDGF to inhibit proliferation and invasion of NSCLC cells. Tumour Biol, 35, 8861-6.

Guo S, Liu HD, Liu YF, et al (2014b). Hepatoma-derived growth factor: a novel prognostic biomarker in intrahepatic cholangiocarcinoma. Tumour Biol, 36, 353-64

Guo Z, He Y, Wang S, et al (2011). Various effects of hepatomaderived growth factor on cell growth, migration and invasion of breast cancer and prostate cancer cells. Oncol Rep, 26, 511-7.

Han Y, Zhang W, Liu Y (2013). Identification of hepatomaderived growth factor as a potential prognostic and diagnostic marker for extrahepatic cholangiocarcinoma.
World J Surg, 37, 2419-27.

Hanada S, Kakehashi A, Nishiyama N, et al (2013). Myristoylated alanine-rich C-kinase substrate as a prognostic biomarker in human primary lung squamous cell carcinoma. Cancer Biomark, 13, 289-98.

Hanahan D (2014). Rethinking the war on cancer. Lancet, 383, 558-63.

Hayes DF, Isaacs C, Stearns V (2001). Prognostic factors in breast cancer: current and new predictors of metastasis. $J$ Mammary Gland Biol Neoplasia, 6, 375-92.

Higgins JP, Thompson SG, Deeks JJ, et al (2003). Measuring inconsistency in meta-analyses. BMJ, 327, 557-60.

Hsu SS, Chen CH, Liu GS, et al (2012). Tumorigenesis and prognostic role of hepatoma-derived growth factor in human gliomas. J Neurooncol, 107, 101-9.

Hu TH, Huang CC, Liu LF, et al (2003). Expression of hepatomaderived growth factor in hepatocellular carcinoma. Cancer, 98, 1444-56.

Hu TH, Lin JW, Chen HH, et al (2009). The expression and prognostic role of hepatoma-derived growth factor in colorectal stromal tumors. Dis Colon Rectum, 52, 319-26.

Iwasaki T, Nakagawa K, Nakamura H, et al (2005). Hepatomaderived growth factor as a prognostic marker in completely resected non-small-cell lung cancer. Oncol Rep, 13, 1075-80.

Li D, Han Z, Liu J, et al (2013a). Upregulation of nucleus HDGF predicts poor prognostic outcome in patients with penile squamous cell carcinoma bypass VEGF-A and Ki-67. Med Oncol, 30, 702.

Li M, Shen J, Wu X, et al (2013b). Downregulated expression of hepatoma-derived growth factor (HDGF) reduces gallbladder cancer cell proliferation and invasion. Med Oncol, 30, 587.

Li SZ, Zhao YB, Cao WD, et al (2013c). The expression of hepatoma-derived growth factor in primary central nervous system lymphoma and its correlation with angiogenesis, proliferation and clinical outcome. Med Oncol, 30, 622.

Lin YW, Li CF, Chen HY, et al (2012). The expression and prognostic significance of hepatoma-derived growth factor in oral cancer. Oral Oncol, 48, 629-35.

Liu YF, Zhao R, Guo S, et al (2011). Expression and clinical significance of hepatoma-derived growth factor as a prognostic factor in human hilar cholangiocarcinoma. Ann Surg Oncol, 18, 872-9.

Mao J, Xu Z, Fang Y, et al (2008). Hepatoma-derived growth factor involved in the carcinogenesis of gastric epithelial cells through promotion of cell proliferation by Erk $1 / 2$ activation. Cancer Sci, 99, 2120-7.

Natrajan R, Williams RD, Hing SN, et al (2006). Array CGH profiling of favourable histology Wilms tumours reveals novel gains and losses associated with relapse. J Pathol, 210, 49-58.

Ordonez-Moran P, Huelsken J (2014). Complex metastatic niches: already a target for therapy? Curr Opin Cell Biol, 31, 29-38.

Parmar MK, Torri V, Stewart L (1998). Extracting summary statistics to perform meta-analyses of the published literature for survival endpoints. Stat Med, 17, 2815-34.

Ren H, Chu Z, Mao L (2009). Antibodies targeting hepatomaderived growth factor as a novel strategy in treating lung cancer. Mol Cancer Ther, 8, 1106-12.

Ren H, Tang X, Lee JJ, et al (2004). Expression of hepatomaderived growth factor is a strong prognostic predictor for patients with early-stage non-small-cell lung cancer. J Clin Oncol, 22, 3230-7.

Savola S, Klami A, Tripathi A, et al (2009). Combined use of expression and $\mathrm{CGH}$ arrays pinpoints novel candidate genes in Ewing sarcoma family of tumors. BMC Cancer, 9, 17. 
Song Y, Hu Z, Long H, et al (2014). A complex mechanism for HDGF-mediated cell growth, migration, invasion, and TMZ chemosensitivity in glioma. $J$ Neurooncol, 119, 285-95.

Tao F, Ye MF, Sun AJ, et al (2014). Prognostic significance of nuclear hepatoma-derived growth factor expression in gallbladder cancer. World J Gastroenterol, 20, 9564-9.

Tierney JF, Stewart LA, Ghersi D, et al (2007). Practical methods for incorporating summary time-to-event data into metaanalysis. Trials, $\mathbf{8}, 16$.

Tsai HE, Liu GS, Kung ML, et al (2013). Downregulation of hepatoma-derived growth factor contributes to retarded lung metastasis via inhibition of epithelial-mesenchymal transition by systemic POMC gene delivery in melanoma. Mol Cancer Ther, 12, 1016-25.

Uyama H, Tomita Y, Nakamura H, et al (2006). Hepatomaderived growth factor is a novel prognostic factor for patients with pancreatic cancer. Clin Cancer Res, 12, 6043-8.

Wang L, Jiang Q, Hua S, et al (2014). High nuclear expression of HDGF correlates with disease progression and poor prognosis in human endometrial carcinoma. Dis Markers, 2014, 298795.

Wang S, Fang W (2011). Increased expression of hepatomaderived growth factor correlates with poor prognosis in human nasopharyngeal carcinoma. Histopathology, 58, 217-24.

Wells GA, Shea B, O'Connell D, et al (2012). The newcastleottawa scale (NOS) for assessing the quality of nonrandomised studies in meta-analyses [Online]. ottawa health research institute web site. http://www.ohri.ca/programs/ clinical_epidemiology/oxford.asp.

Woodward M (2014). A consensus plan for action to improve access to cancer care in the association of Southeast Asian nations (ASEAN) region. Asian Pac J Cancer Prev, 15, 8521-6.

Yamamoto S, Tomita Y, Hoshida Y, et al (2007). Expression level of hepatoma-derived growth factor correlates with tumor recurrence of esophageal carcinoma. Ann Surg Oncol, 14, 2141-9.

Yamamoto S, Tomita Y, Hoshida Y, et al (2006). Expression of hepatoma-derived growth factor is correlated with lymph node metastasis and prognosis of gastric carcinoma. Clin Cancer Res, 12, 117-22.

Yang Y, Li H, Zhang F, et al (2013). Clinical and biological significance of hepatoma-derived growth factor in Ewing's sarcoma. J Pathol, 231, 323-34.

Yang Y,Zhen T, Zhang F, et al (2014).p53 and hepatoma-derived growth factor expression and their clinicopathological association with Ewing family tumour. J Clin Pathol, 67, 235-42.

Ye F, Cheng Q, Zhou C, et al (2011). Hepatoma-derived growth factor is a novel prognostic factor for cervical squamous cell carcinoma. Histopathology, 58, 982-4.

Yoshida K, Tomita Y, Okuda Y, et al (2006). Hepatoma-derived growth factor is a novel prognostic factor for hepatocellular carcinoma. Ann Surg Oncol, 13, 159-67.

Zhang A, Long W, Guo Z, et al (2010). Development and clinical evaluation of a multi-purpose mAb and a sandwich ELISA test for hepatoma-derived growth factor in lung cancer patients. J Immunol Methods, 355, 61-7.

Zhang J, Chen N, Qi J, et al (2014). HDGF and ADAM9 are novel molecular staging biomarkers, prognostic biomarkers and predictive biomarkers for adjuvant chemotherapy in surgically resected stage I non-small cell lung cancer. $J$ Cancer Res Clin Oncol, 140, 1441-9.

Zhang J, Qi J, Guo Y, et al (2011). Aberrant expression of HDGF and its prognostic values in surgically resected non-small cell lung cancer. Chinese J Lung Cancer, 14, 211-8.
Zhao J, Yu H, Lin L, et al (2011). Interactome study suggests multiple cellular functions of hepatoma-derived growth factor (HDGF). J Proteomics, 75, 588-602.

Zhou NX, Zhou YY, Huo JR (2010a). Significance of HDGF expression in hepatocellular carcinoma tissue and HepG2 cells. World Chinese J Digestol, 18, 2427-33.

Zhou S, Xu S, Zhang H, et al (2007a). Expression of hepatomaderived growth factor and its clinical implication in stage I non-small cell lung cancer. Chinese J Lung Cancer, 10, 291-5.

Zhou SJ, Xu SF, Zhang HQ, et al (2007b). Expression of HDGF and its implication in stage I non-small cell lung cancer. Chinese J Oncol, 29, 927-30.

Zhou Y, Zhou N, Fang W, et al (2010b). Overexpressed HDGF as an independent prognostic factor is involved in poor prognosis in Chinese patients with liver cancer. Diagn Pathol, 5, 58 . 